\title{
Artifact Removal from Skin Dermoscopy Images to Support Automated Melanoma Diagnosis
}

\author{
Dr. Ahlam Fadhil Mahmood \\ ahlam.mahmood@gmail.com \\ Hamed Abdulaziz Mahmood \\ Computer Engineering Department \\ hamedce43@gmail.com \\ University of Mosul
}

\begin{abstract}
:-
The main challenge in an automated diagnostic system for the early diagnosis of melanoma is the correct segmentation. In skin dermoscope images many artifacts such as ruler markings, air bubbles and hairs must be removed to correctly diagnosis skin cancer. This paper focuses on the use of image processing techniques to automatically detects and removes hairs and ruler markings from dermoscopy images. The proposed algorithm includes two main steps: firstly, hairs and ruler marking are isolated by generating a binary image mask include these artifacts only. The suggested mask procedure start with separate RGB dermoscopy images to the red, green and blue color components. Utilizing red channel to create the mask by applying noise removing on this plan, then adaptive canny edge detector is used for refinement by morphological operators. Secondly, the white regions of the mask are repaired based on polygons inpainting . Experiment on a number of dermoscopy images demonstrates that the proposed method produces superior results compared to existing techniques.
\end{abstract}

Keywords: Skin Cancer, Dermoscopy, Artifact Removal, Inpainting

$$
\begin{aligned}
& \text { إزالة معالم مؤثرة من الصور المجهرية للجلد للتمكن من التشخيص الآلي للاورام }
\end{aligned}
$$

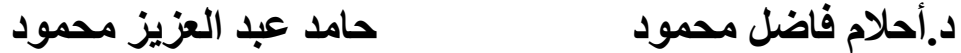

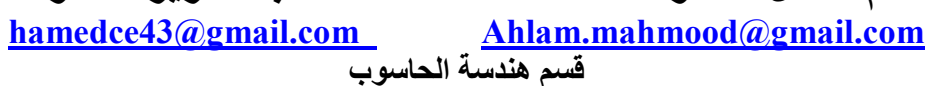

$$
\begin{aligned}
& \text { جامعة الموصل الحلب }
\end{aligned}
$$

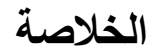

أن التحدي الرئيسي في نظام التثخيص الآلي المبكر لسرطان الجلا هو التجزئة الصحيحة. فقي صور الجلا يوجد

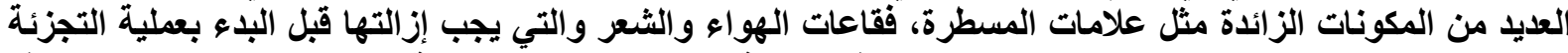

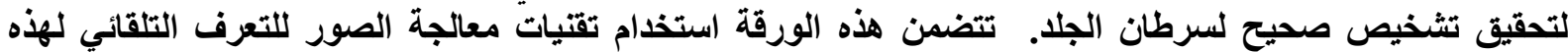

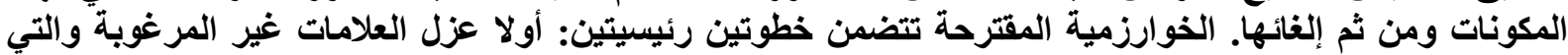

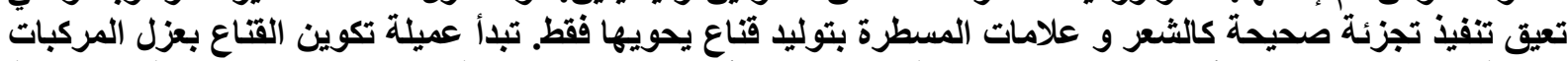

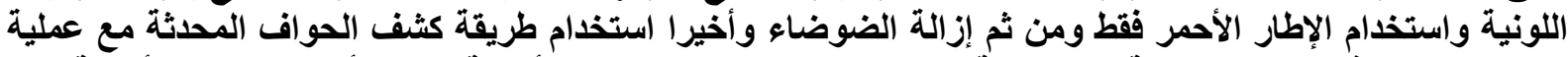

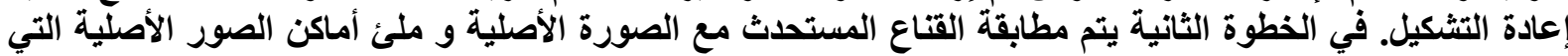

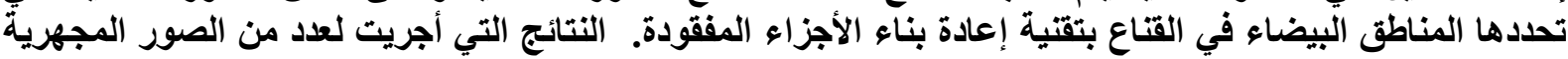

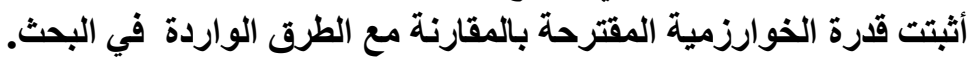




\section{Introduction}

For Years, cancer has been one of the biggest threats to human life; it is expected to become the leading cause of death over the next few decades. Based on statistics from the World Health Organization (WHO) [1]; deaths caused by cancer are expected to increase in the future, with an estimated 12 million people dying from cancer in 2030 [1]. Of all the known cancers, skin cancer is a major concern in both developed and developing countries over the past 40 years, and its incidence has increased in recent years. Melanoma is expected to result in over 65,000 deaths in 2013 [2], but the survival rate is quite high if the cancer is detected early. Hence, the early detection of malignant skin lesions is critical to preventing death[3][4].

Dermoscopy has become important technique in early diagnosis of melanoma. In this technique, oil is applied on skin surface where lesion is present and polarized light is made incident on skin. Then image is acquired with digital camera attached to dermatoscope. This process reveals the morphological structures which are present in deeper layer of skin[3].

When image acquisition is done using dermatoscope, some artifacts are introduced in image. In some of the cases, dermatoscope is provided with ruler markings for measurement of diameter of lesion. The air bubbles and hair in image can affect the accuracy of segmentation process and further diagnosis of skin cancer. So these artifacts must be removed from dermoscopic images[4].

This paper is organized as follows: Section 2 discusses the different image processing techniques used for removing various artifacts in dermoscopic image. Section 3 gives details of the proposed hair detection and removing techniques used for artifact removal from dermoscopy images. In section 4, Experimental Results are provided for detection and repairing used methods. Then paper is concluded with discussion in section 5 .

\section{Literature Survey}

Various researches have been pursued in hair detection and remove for a number of years and still the area is being explored. Hair occlusion can cause segmentation algorithms to perform badly due to confusion between hair and mole borders. Hence, the removal of hair is an important pre-processing step in such systems.

The most popular hair detection method is DullRazor [5]. DullRazor applies a grayscale morphological closing operation with a linear structuring element to detect dark hair. The algorithm consists of three basic steps: Identifying the dark hair locations by morphological closing operation, replacing the hair pixels by bilinear interpolation, and smoothing the final result by adaptive median filter. The goal of DullRazor's method is removing dark thick hairs, thus it cannot remove light colored or thin hairs [3].

K. Kiani and A. R. Sharafat suggest an improved of DullRazor algorithm first detect the predominant orientation of hairs in the image by using Radon transform, followed by filtering the image by Prewitt filters using the orientation of existing hairs. Their proposed scheme has the advantage of removing bubbles, as mentioned in[6].

Schmid saugeon et al. used a similar approach but the morphological closing operator is applied to the three components of the LUV color space. The accuracy of the proposed method was not reported in this research and it has similar applicability limitations as DullRazor[7].

Zhou et al. implemented automatic hair and ruler marking detection using curvilinear structure analysis and performed explicit curve fitting to increase the robustness of their detection algorithm. Finally, the artifact pixels were replaced by a feature guided exemplarbased inpainting method. This algorithm is applicable to dark hair only[8]. 
In the literature on hair removal, a few papres used the morphological closing operator to enhance hair and applied a statistical threshold to detect the hair regions utilized the concept of non-linear-PDE based diffusion [9][10].

Abbas et al. proposed novel hair detection and repairing algorithm. Hairs are detected by a derivative of Gaussian method and subsequently enhanced by a morphological technique which are inpainted by a fast marching method[11].

M. Toossi et al., the proposed algorithm includes two steps: firstly, morphological operators segment light and dark hairs and ruler marking through adaptive canny edge detector and refinement. Secondly, the hairs are repaired based on multi-resolution coherence transport inpainting using a wavelet based structure[3].

P. R. Mahajan and A. J. Vyavahare enhance a contrast limited applying adaptive histogram equalization (CLAHE) approach that improves the contrast between skin and lesion. Also two hair removal methods based on morphological closing and top hat transformation combined with bicubic interpolation has been implemented[4].

This article, presents an algorithm to segment both dark and light colored hairs with any thickness and ruler marking, and remove them from dermoscopy images.

\section{The proposed algorithm}

The proposed hair removal algorithm consists of two steps: (1) Artifact detection with the use of adaptive canny edge detector and refinement by morphological operators and (2) Hair repair by inpainting technique.

\section{1- Artifact Detection from Dermoscopy Images}

The database images are obtained from different sources, so the images size is non-standard and the preprocessing was firstly done as illustrated in the flow chart that is shown in Figure 1.

Firstly, resize all images to have same size $(512 \times 512)$ pixels. Then most dermoscopy images often contain black corners, which also must be removed because they might interfere with the subsequent border detection steps. In order to determine the darkness of a pixel with ( $R, G$, and $B$ ) coordinates, the lightness component is utilized[12]:

$\mathrm{L}=\frac{\max (R, G, B)+\min (R, G, B)}{2}$

In particular, a pixel is considered black if its lightness value is $<20$ in equation (1). Using this criterion, the image is scanned row-by-row starting from the top. A particular row is labeled as part of the black frame if it contains $60 \%$ black pixels. The top-to-bottom scan terminates when a row that contains less than the threshold percentage of pixels is encountered. The same scanning procedure is repeated for the other three main directions.

After preprocessing steps, due to the simplicity computation

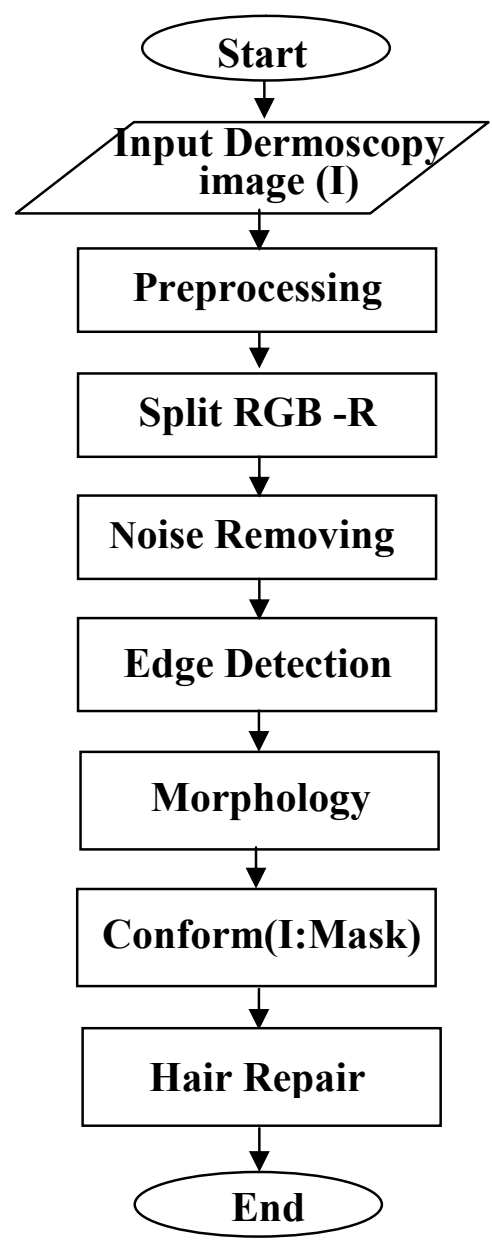

Figure 1: Flow Chart of the Proposed Algorithm 
of singal channel compared with three RGB channels. Each RGB image is often converted to a scalar image using one of the following methods:

- Retaining only the red channel (lesions are often more prominent in this channel).

- Applying the luminance transformation, i.e.

$$
Y=0.299 R+0.587 G+0.114 B
$$

- Applying the Principal Component Analysis[3] and retaining the channel with the highest variance.

Associating chromophores absorbance with the spectral responses of conventional RGB cameras, the image intensity in red channel is $(\sim 650 \mathrm{~nm})$, while those equal to $(\sim 550 \mathrm{~nm})$, $(\sim 450 \mathrm{~nm})$ of the green and blue channels. So utilizing only red channel of RGB image to separate the hairs and rulers from the dermoscope images [13].

For noise removing, a $3 \times 3$ pixels wiener filter was used. The wiener mask size is very small compared with the size of the images to preserve the edges in the image.

After filtering, an improved canny edge detection method is used for detecting the boundaries of hairs [3], [14]. The traditional canny edge detector gets the high threshold from the gradient cumulative histogram, therefore when the noise density increases, some noise pixels may be calculated as edge points. Rosenfeld et al. proposed an image threshold segmentation method based on histogram concavity analysis; therefore, this method is used to select the dual threshold [3]. The method is based on obtaining the convex hull of the gradient magnitude histogram and analyzing the concavities of convex hull. When the convex hull is calculated, the deepest concavity points become candidates as high threshold (Th) for canny operator and the low threshold is equal $0.5 \times \mathrm{Th}$.

The boundaries of hairs are detected by adaptive canny edge detection and the hairs are segmented by applying the morphological dilation operator on the edge-detected image. That is performed by laying the structuring element on the image and sliding it across the image in a manner similar to convolution. It can be noticed that segmented image contained misclassified pixels, which may be related to lesion pigment network or skin lines. The length of many unwanted segmented objects is usually less than the length of normal hairs. Considering the fact that hairs are thin and long, special labeling and morphological operations are utilized to detect them. Lines structuring elements oriented in different directions perform morphological opening. Eventually, to obtain smooth hair lines, dilation and filling operators are applied to the hair mask. By removing unwanted objects from hair mask, hair and ruler mask result is obtained as illustrated in Figure 2 for different cases.

\section{2- Artifact Repair}

The effect of artifacts on diagnosis analysis can be reduced by replacing them with patches which are similar to the neighboring pixels. Image inpainting is used for removing and repairing unnecessary elements such as hairs and ruler marks from images. M. Toossi et al. proposed fast image inpainting based on wavelet structure. It combines the simple coherence transport inpainting with a wavelet decomposition/reconstruction method in an iterative and multi-resolution structure[3][15].

In this paper, after the artifact binary mask was extracted by a previous procedure, a simple image inpainting technique is performed to removing unwanted objects from the original dermoscopy image by diffusing the pixel information from the neighbourhood pixels. The image is separated into two images, the real Dermoscopy image and mask images, the area to be inpainted is marked with white pixel in the binary mask. When the inpainted region are selected, the real image is painted with skin neighboring color pixels using concave polygon edge with the fence are identified. A single pixel in each region is chosen and inside-outside tests are carried on it. If the test returns interior, the pixel is colored. Otherwise, the pixel remains uncolored[16]. This method is easier and more effective than the method polygons. 


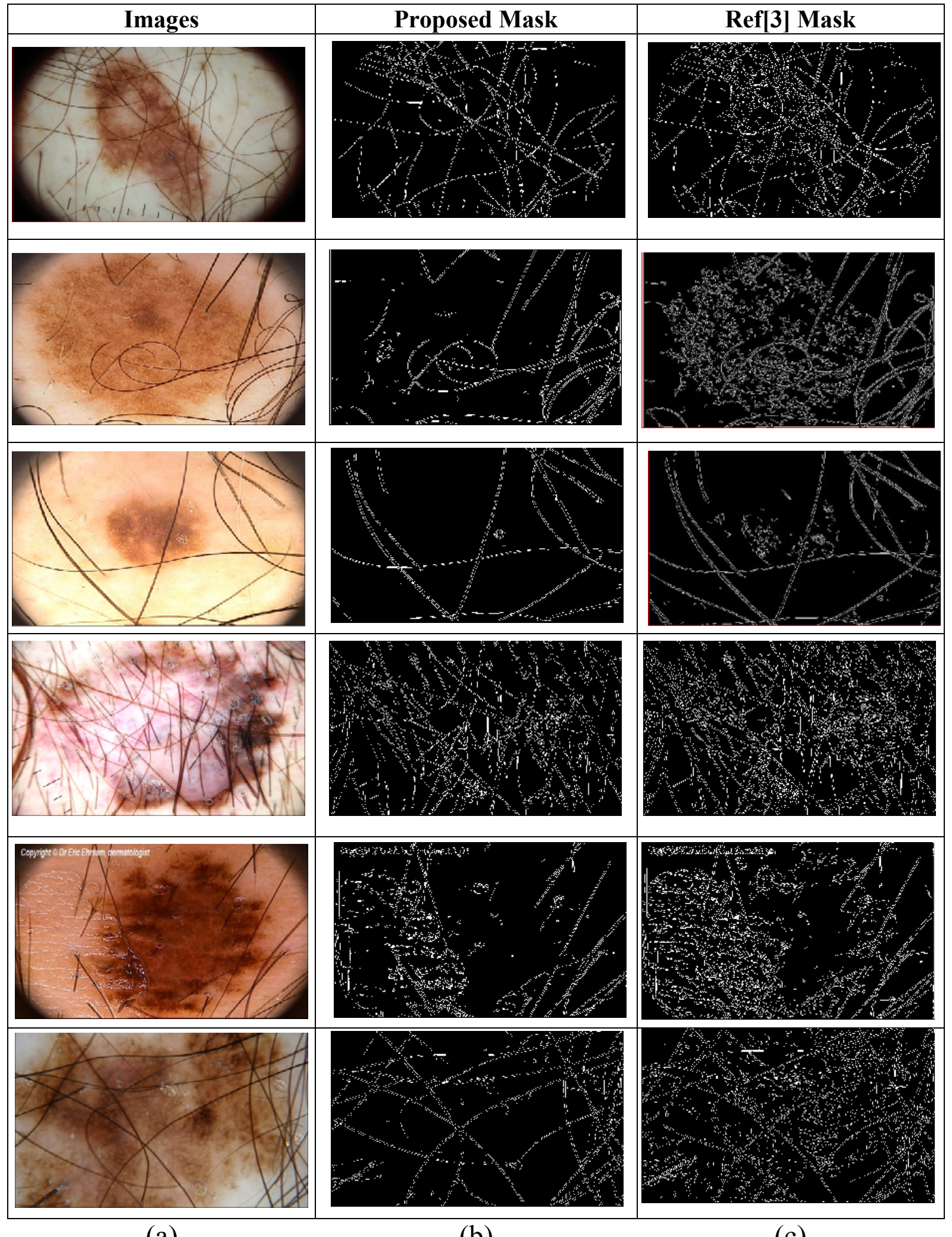

(a)

(b)

(c)

Figure 2: a ) Original dermoscopic image with rulers markings and hairs

b) Proposed binary Hair, air bubbles and rulers mask obtain

c) Artifacts binary mask in Ref[3] 
A fence location is usually considered passing through any polygon vertex for simplicity. A bounding rectangle for the polygon is constructed. The regions bounded by the present in the previous work, as it is not depend on the automatic threshold value and the thickness of hair strands.

\section{Experimental Results}

The proposed method was tested on a set of 80 dermoscopy images acquired from atlases of dermoscopy http://www2.fc.up.pt/addi and http://www.dermoscopic.blogspot.com . These images obtained from different sources and stored in the RGB color format with dimensions ranging from $689 \times 554$ pixels to $768 \times 560$ pixels. Manual hair segmentation given by dermatologist is used as ground truth for the performance evaluation. The proposed method was developed in MATLAB version R2013a (8.1.0.604).

\subsection{Objective Result of The Proposed Detection Method}

To estimate the accuracy of the proposed artifact detect algorithm and to quantify the automatic hair detection error, quantitative evaluations were performed using three statistical metrics: True Detection Rate (TDR), False Positive Rate (FPR), and Diagnostic Accuracy (DA).

TDR measures the rate of pixels which were classified as hair by both the automatic algorithm and the medical expert, and FPR measures the rate of pixels which were classified as hair by the automatic segmentation and were not classified as hair by the medical expert. These metrics are calculated as follows:

$$
\begin{aligned}
& \text { True Detection Rate }(T D R)=\frac{T P}{T P+F N} \times 100 \% \\
& \text { False Positive Rate }(F P R)=\frac{F P}{T P+F N} \times 100 \% \\
& \text { Diagnostic Accuracy }(D A)=\frac{T P}{T P+F P+F N} \times 100 \%
\end{aligned}
$$

Where TP, FP, and FN stand for the number of true positive, false positive, and false negative, respectively. These metrics are computed to compare the proposed hair detection algorithm with the DullRazor hair removal software[17], the hairs are repaired based on multi-resolution coherence transport inpainting[3].

The quantitative results of the proposed algorithm, DullRazor software, multi-resolution coherence software and bicubic interpolation are presented in Table 1. It can be noticed that the proposed algorithm achieves high DA of $90.7 \%(\mathrm{TDR}=96.1 \%$ and FPR $=1 \%)$, whereas others methods achieves less quality.

Table 1: Comparison of the hair detection algorithms

\begin{tabular}{|l|c|c|c|}
\hline Artifact Detection Method & DA(\%) & TDR(\%) & FPR(\%) \\
\hline The Proposed algorithm & 90.7 & 96.1 & 1 \\
\hline Multi-Resolution[3] & 88.3 & 93.2 & 4 \\
\hline DullRazor[17] & 48.6 & 70.2 & 33.4 \\
\hline
\end{tabular}

\subsection{Subjective Result of the Proposed Hair Repaired Method}

The performance of the proposed polygon edge in painting method is presented in Figure 3. As showing in figure, the database of images including wide variety of cases that is include label, hairs, air bubbles and rulers marking. The results indicate that the proposed algorithm is highly accurate and able to detect and inpaint all these arifacts pixels with few errors. 

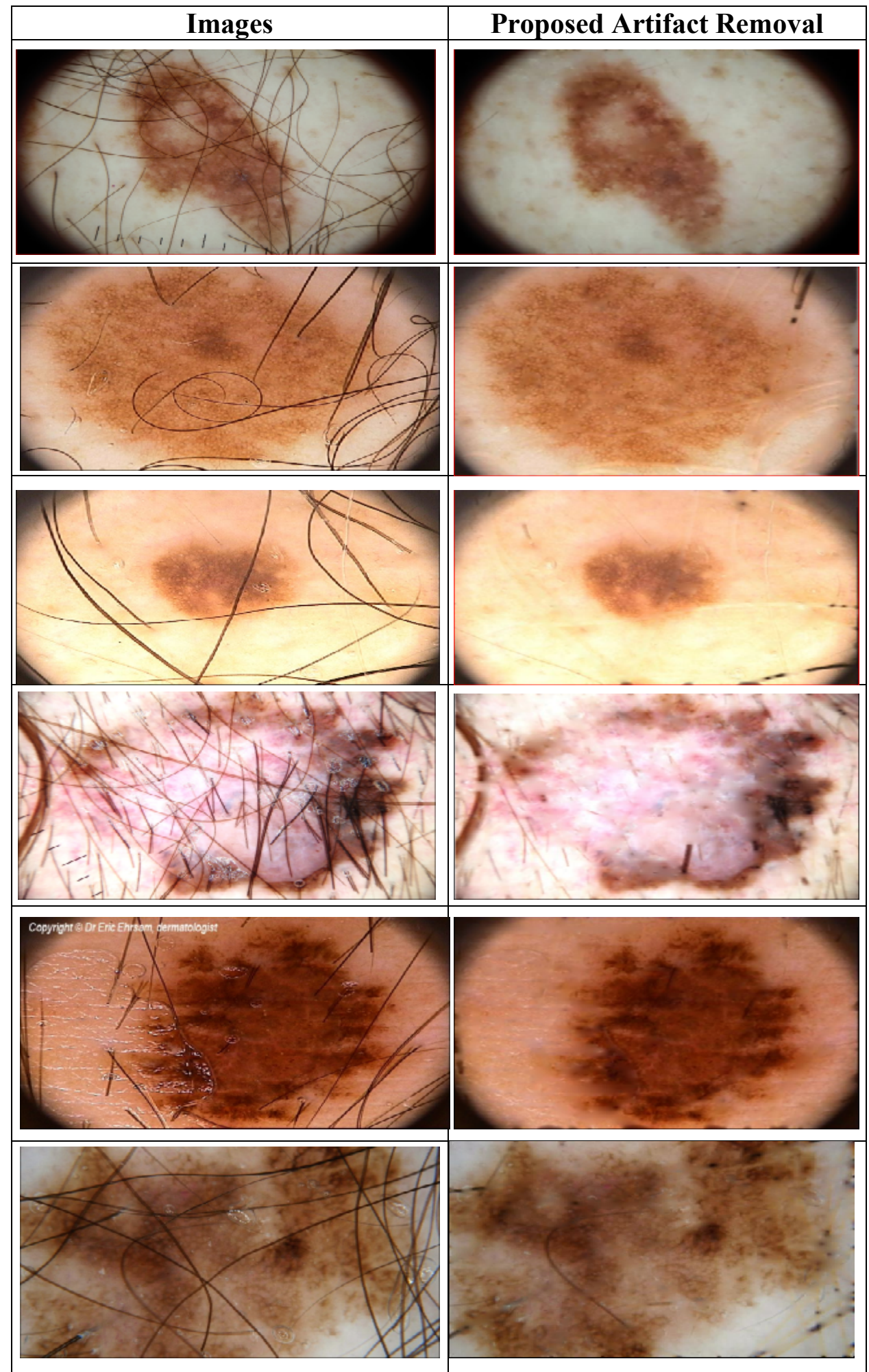

(a)

(b)

Figure 3: a) Original dermoscopic images with ruler, air bubbles, Lable and hairs

b) Artifact removal image 


\subsection{Segmentation After Artifact Removal}

To evaluate skin lesion segmentation, the manual segmentations are carried out on the original images by some dermatologists. Then, images that their containing hairs are detected and repaired by the proposed algorithm are segmented automatically using the region-based active contour method[18]. The percentage of segmentation error is calculated by comparing the results of manual and automatic segmentations using the following equation[3]:

Segmentation Error $=\frac{\operatorname{Area}(A S \oplus M S)}{\operatorname{Area}(M S)} \times 100 \%$

where AS and MS are the binary images obtained by automatic and manual segmentation, respectively, and $\oplus$ is the logic exclusive-OR operation. Table 2 shows the segmentation error on the images before and after hair removal. It can be seen that the segmentation error is effectively reduced after hair removal. Specially, skin lesion segmentation after the proposed hair detection and hair-repairing algorithm has minimum error with respect to other cases.

Table 2.The Results of Segmentation after and before artifact removal algorithm.

\begin{tabular}{|c|c|c|}
\hline Artifact Detection Method & Artifact Repairing Method & Segmentation Error(\%) \\
\hline None & None & 16.26 \\
\hline DullRazor & DullRazor & 12.11 \\
\hline$[11]$ & Coherence transport & 10.74 \\
\hline$[3]$ & multi-resolution coherence transport & 9.9 \\
\hline The Proposed & polygons & 8.6 \\
\hline
\end{tabular}

\section{Conclusion}

This paper presents artifact removal from dermoscopic images which are useful for early diagnosis of melanoma. The automatic hair, label, air bubble and ruler marking detection and removing scheme are achieved in the proposed algorithm. After the artifact pixels are selected from white region in binary mask, replace them using polygon-based inpainting procedure. This algorithm was tested on a data set of 80 dermscopy images and the results are promising; it is fastest due to operate in red channel only and it is simplest approaches in comparison to existing techniques.

\section{References}

[1]American Cancer Society, Cancer Facts \& Figures 2012. Atlanta: American Cancer Society, 2012.

[2]Amercian Academy of Dermatology, Skin Cancer [Online]. Available: http://www.aad.org/media-resources/stats-and-facts/conditions/skin-cancer.

[3]M. T. Bahreyni Toossi, H. R. Pourreza., H. Zare, M. H Sigari, P. Layegh and A. Azimi," An effective hair removal algorithm for dermoscopy images", Skin Research and Technology; Published by John Wiley \& Sons Ltd, 19: PP.230-235, 2013.

[4]P. R. Mahajan and A. J. Vyavahare, "Artifact Removal and Contrast Enhancement for Dermoscopic Images Using Image Processing Techniques", International Journal of Innovative Research in Electrical, Electronics, Instrumentation and Control Engineering Vol.1, Issue 9, PP. 418- 421, December 2013. 
[5]P. Wighton, T. K. Lee, H. Lui, D. I. McLean, and M. S. Atkins, "Generalizing Common Tasks in Automated Skin Lesion Diagnosis", IEEE Transactions on Information Technology in Biomedicine, Vol. 15, No. 4, PP.622-629, July 2011.

[6]K. Kiani and A. R. Sharafat, "An improved DullRazors for digitally removing dark and light-colored hairs in dermoscopic images", Computers in Biology and Medicine 41, PP.139145, 2011.

[7]P. S. Saugeona, J. Guillodb, J. Thirana, "Towards a computer aided diagnosis system for pigmented skin lesions" Computerized medical Imaging and Graphics ; 27: PP. 65-78, 2003.

[8]H. Zhou, M. Chen, R. Gass, J. M. Rehg, "Feature-preserving Artifact Removal from Dermoscopy Images", In Proceeding of SPIE Medical Imaging Conference; 6914: 69141B1,San Diego, 16 February, 2008.

[9]C. Bibiane, "Modern PDE Techniques for Image Inpainting", Ph.D thesis, DAMTP, Centre for Mathematical Sciences University of Cambridge, 15th of June 2009.

[10]F. Bornemann and T. März, "Fast image inpainting based on coherence transport", J Math Imaging Vis; 28: PP. 259-278, 2007.

[11]Q. Abbas, M.E. Celebi, I. F. Gari'a, "Hair removal methods: A comparative study for dermoscopy images", Biomedical Signal Processing and Control 6, PP.395-404, 2011.

[12]V. Powar, A. Jahagirdar and S. Sirsikar, "Skin Detection in YCbCr Color Space", International Journal of Computer Applications (0975 - 8887) Volume *_ No.* 2011.

[13]A. B. Afonso, "Classification of Pigmented Skin Lesions based on Color Features", MSc. Thesis of Biomedical Engineering, Lisboa University, 2013.

[14] Rashmi , M. Kumar, A. K.Jaiswal and R. Saxena, "Performance Analysis of Adaptive Canny Edge Detector Using Bilateral Filter", International Journal of Innovative Research in Computer and Communication Engineering, Vol. 1, Issue 4, June 2013.

[15]R. Biradar and V. Vkohir, "A novel image inpainting technique based on median diffusion", Sadhana Vol. 38, Part 4, pp. 621-644, Indian Academy of Sciences, August 2013,. [16]S.Anitha and D.Evangeline," An Efficient Fence Fill Algorithm using Inside-Outside Test", nternational Journal of Advanced Research in Computer Science and Software Engineering, ISSN: 2277 128X, Volume 3, Issue 11, pp. 605-609, November 2013.

[17]P. Wighton, T. K. Lee and M. S. Atkins, " Dermascopic hair disocclusion using inpainting", Medical Imaging, Proc. of SPIE Vol. 6914, 691427, PP1-8, 2008.

[18]K. Zhang, L. Zhang, H. Song, W. Zhou, "Active contours with selective local or global segmentation: A new formulation and level set method", Image and Vision Computing 28 , PP. 668-676, 2010. 